\title{
Elektrophoretische Untersuchungen zur Organspezifität der wasserlöslichen Proteine in der Ontogenese von Drohnen (Apis mellifera L.)
}

\author{
Evgenija IvANOvA \\ Paissij-Hilendarski Universität Plovdiv, Biologische Fakultät, \\ Sektion Genetik. Zar Assenstr. 24, Plovdiv, Bulgarien
}

(Received 30 October 1999; revised 15 March 2000; accepted 20 July 2000)

\begin{abstract}
Zusammenfassung - Wasserlösliche Proteine aus Organextrakten von Herz, Darm, Hoden, Mucusdrüsen und Augen von Drohnen wurden im Verlauf ihrer Ontogenese durch Elektrophorese in Polyacrylamidgel in individuellen Proben untersucht. In keinem einzigen der untersuchten Organe treten die insgesamt 31 nachgewiesenen Eiweißfraktionen im Verlauf der Individualentwicklung gleichzeitig auf. Die Anzahl der Fraktionen in den Organen ist unterschiedlich: im Herzextrakt fanden wir 22, im Darm 24, im Hodenextrakt 21, im Extrakt der Mucusdrüse 20 und im Augenextrakt 16 Fraktionen. Einige Proteinfraktionen weisen eine klare Stadien- und Organspezifität auf, andere sind in mehreren Entwicklungsstadien ausgeprägt.
\end{abstract}

Apis mellifera L. / Drohnen / Ontogenese / wasserlösliche Proteine

\section{EINLEITUNG}

Bei der Untersuchung molekularer Grundlagen der Entwicklung der Organismen nehmen ontogenetische Untersuchungen der Eiweißsysteme eine wesentliche Stellung ein. Frühere Untersuchungen der wasserlöslichen Eiweiße in Haemolymphe und Totalextrakt zeigen eine Dynamik ihrer Ausprägung im Verlauf der Individualentwicklung der Honigbienen [1-4, 7, 8, 11, $14,16]$.
Außer quantitativen Angaben über die Proteine gibt es auch qualitative Untersuchungen, vor allem bei globulin- und albuminähnlichen Anteilen in der Haemolymphe der Bienen $[5,15]$. Außerdem wurden die Proteine des Mitteldarms [15] und der Hypopharyngialdrüsen charakterisiert $[6,9$, 12, 13].

Die vorliegende Arbeit untersucht das Auftreten von wasserlöslichen Proteinen bei Drohnen von Apis mellifera L. und bestimmt durch vergleichende Analyse ihre Organspezifität im Verlauf der Ontogenese. 


\section{MATERIAL UND METHODEN}

Bei der elektrophoretischen Analyse untersuchten wir 368 Organproben von einzelnen Drohnen. Das Material wurde in der Zeit von 1997-1998 gesammelt und bearbeitet.

Folgende Ontogenesephasen wurden untersucht: 5 Tage alte Rundmaden (nicht verdeckelt), 7 Tage alte Streckmaden (verdeckelt), 3 Tage alte weißäugige und 7 Tage alte dunkeläugige Puppen und 10 Tage alte Imagines.

Nach der Isolierung der Organe Herz, Darm, Mucusdruse, Hoden und Augen unter einer Binokularlupe wurden sie mehrmals mit destilliertem Wasser gewaschen, um den Fettkörper zu entfernen. Der Darm wurde vom Darminhalt gereinigt. Danach extrahierten wir das Material 24 Stunden in einem $0,1 \mathrm{M}$ Tris-Phosphatpuffer, $\mathrm{pH}$ 6,7 bei einer Temperatur von $4{ }^{\circ} \mathrm{C}$. Nach der Zentrifugation bei $900 \mathrm{~g}$ für 30 Minuten wurde der Überstand nicht zusätzlich konzentriert sondern in verdünnter nativer Form benutzt.

Die elektrophoretische Trennung der Organextrakte führten wir nach dem ersten System von Maurer [10] in 7,5\% Trenngel und 3,3\% konzentrierendem Gel durch, die Kapazität einer Gelplatte betrug 23 Proben. Die Trennung verlief in zwei Puffersystemen, einem Tris Chlorid- $(\mathrm{pH} \mathrm{8,9)}$ und einem Tris Phosphatsystem ( $\mathrm{pH} \mathrm{6,7).}$

Der Anoden- und Kathodenpuffer für die Kammern war 0,05M Tris- 0,2M Glycin mit pH 8,3.

Die Elektrophorese wurde bei $2-4{ }^{\circ} \mathrm{C}$ durchgeführt und benötigte etwa 3 Stunden bei einer Spannung von 200 V im konzentrierenden Gel und $400 \mathrm{~V}$ im Trenngel.

Die Färbung der Proteine erfolgte mit Coomassie-Brillantblau R 250 in $14 \%$ iger Trichloressigsäure in etwa 2 Tagen, danach wurden die Platten in $7 \%$ iger Essigsäure aufbewahrt.

\section{ERGEBNISSE}

Die insgesamt auftretenden 31 wasserlöslichen Proteinfraktionen kennzeichneten wir entsprechend ihrer Laufgeschwindigkeit mit den Buchstaben von A bis ZE. Das unterschiedliche Auftreten der Fraktionen nach Organen und Altersstufen ist in Tabelle I und Abbildungen 1 und 2 zusammengefa $\beta$ t. Diese Daten wurden zu zwei Vergleichsanalysen der wasserlöslichen Eiweiße bei Drohnen verwendet: 1 . Unterschiede bei den Organen und 2. Unterschiede im Verlauf der Ontogenese.

\subsection{Herz}

Im Madenstadium und beim Imago besteht das Eiweißspektrum im Herzextrakt aus einer bedeutend größeren Anzahl von Fraktionen $(\mathrm{N}=13$ bzw. 16) als im Vorpuppen- und Puppenstadium ( $\mathrm{N}=6$ bzw. 1 , Tab. I). Einige Fraktionen treten nur während spezieller Entwicklungsstadien auf. Bei Rundmaden sind es die Fraktionen F, $\mathrm{O}$ und $\mathrm{T}$ und bei Streckmaden die langsam wandernden Fraktionen ZA, ZB und ZE. Bei den Imagines (Abb. 1) sind die Fraktionen $\mathrm{B}$ und $\mathrm{C}$ mit hoher und die Fraktion $\mathrm{R}$ mit mittlerer Laufgeschwindigkeit ausgeprägt.

Außer diesen stadienspezifischen Fraktionen treten einige bei mehreren, aber niemals allen Stadien auf. Dies gilt für die Fraktionen D, E, H, J, M, N, Q, S, W, die im Madenstadium erscheinen, im Vorpuppenund Puppenstadium fehlen und im Imagostadium wieder auftreten. Die Fraktionen $\mathrm{Z}$ und ZD, die für Streckmaden und Imagines charakteristisch sind, fehlen bei Rundmaden, wei $\beta$ - und dunkeläugigen Puppen. Beständiges und starkes Auftreten während der gesamten postembryonalen Periode stellten wir nur bei der Eiweißfraktion V fest (Tab. I).

\subsection{Darm}

Auch im Darm ergab sich eine spezifische Stadienausprägung für die folgenden 
Tabelle I. Organspezifität wasserlöslicher Proteine in der Drohnenontogenes: 1-Rundmaden; 2-Streckmaden; 3-weissäugige Puppen; 4-dunkeläugige Puppen; 5-Imago.

+ Auftreten; - Fehlen.

\begin{tabular}{|c|c|c|c|c|c|c|c|c|c|c|c|c|c|c|c|c|c|c|c|c|c|}
\hline \multirow{2}{*}{$\begin{array}{l}\text { Organen } \\
\text { Stadien } \\
\backslash \\
\text { Fraktionen }\end{array}$} & \multicolumn{5}{|c|}{ Herz } & \multicolumn{5}{|c|}{ Darm } & \multicolumn{5}{|c|}{ Hoden } & \multicolumn{3}{|c|}{ Mucusdrüse } & \multicolumn{3}{|c|}{ Augen } \\
\hline & 1 & 2 & 3 & 4 & 5 & 1 & 2 & 3 & 4 & 5 & 1 & 2 & 3 & 4 & 5 & 3 & 4 & 5 & 3 & 4 & 5 \\
\hline A & - & - & - & - & - & - & - & - & - & - & - & + & - & - & - & - & - & + & - & - & - \\
\hline B & - & - & - & - & + & - & + & - & - & - & - & + & - & - & - & - & - & + & - & - & - \\
\hline $\mathrm{C}$ & - & - & - & - & + & - & - & - & - & - & + & + & - & - & - & - & - & + & + & - & - \\
\hline $\mathrm{D}$ & + & - & - & - & + & + & - & - & - & + & + & - & + & + & - & - & - & - & - & + & + \\
\hline $\mathrm{E}$ & + & - & - & - & + & + & + & - & - & + & - & + & - & + & + & - & - & + & - & + & + \\
\hline $\mathrm{F}$ & + & - & - & - & - & - & - & - & - & - & - & + & - & - & - & - & - & + & - & - & - \\
\hline G & - & - & - & - & - & - & - & - & - & - & - & + & - & - & - & - & - & - & - & - & - \\
\hline $\mathrm{H}$ & + & - & - & - & + & + & - & - & - & + & + & + & - & + & - & - & - & + & + & + & - \\
\hline I & - & - & - & - & - & - & - & - & - & - & - & + & + & + & - & - & + & - & - & - & + \\
\hline $\mathbf{J}$ & + & - & - & - & + & + & + & - & - & + & - & + & + & + & + & - & + & + & + & + & + \\
\hline $\mathrm{K}$ & - & - & - & - & - & + & - & - & - & + & - & - & - & - & - & - & - & - & - & - & - \\
\hline $\mathrm{L}$ & - & - & - & - & - & + & - & - & - & - & - & - & - & - & - & - & - & - & - & - & - \\
\hline M & + & - & - & - & + & + & - & - & - & + & - & + & + & + & - & - & + & + & + & + & - \\
\hline $\mathrm{N}$ & + & - & - & - & + & - & - & - & - & + & - & + & + & + & - & - & + & + & + & + & - \\
\hline $\mathrm{O}$ & + & - & - & - & - & + & - & - & - & - & - & + & + & + & - & - & - & - & - & - & - \\
\hline $\mathrm{P}$ & - & - & - & - & - & + & - & - & - & - & - & - & - & - & - & - & - & - & - & - & + \\
\hline $\mathrm{Q}$ & + & - & - & - & + & + & - & - & - & + & + & + & - & + & - & - & - & + & + & + & - \\
\hline $\mathrm{R}$ & - & - & - & - & + & - & + & - & - & + & - & - & - & - & - & - & - & + & - & - & + \\
\hline $\mathrm{S}$ & + & - & - & - & + & + & + & - & - & + & - & + & + & - & - & - & + & + & - & - & - \\
\hline $\mathrm{T}$ & + & - & - & - & - & + & - & - & - & + & + & + & + & + & - & - & + & + & - & - & + \\
\hline $\mathrm{U}$ & - & - & - & - & + & + & + & - & - & + & + & + & + & + & - & - & - & + & - & - & + \\
\hline V & + & + & + & + & + & + & + & - & - & + & + & + & + & + & + & - & + & + & - & - & - \\
\hline W & + & - & - & - & + & - & - & + & - & + & - & + & + & + & - & + & + & + & - & + & - \\
\hline$X$ & - & - & - & - & - & - & - & - & + & - & - & - & - & - & - & - & - & - & - & - & - \\
\hline $\mathrm{Y}$ & - & - & - & - & - & - & - & - & + & - & - & - & - & - & - & - & - & - & - & - & - \\
\hline Z & - & + & - & - & + & + & - & + & + & + & - & + & + & + & + & - & - & + & + & + & + \\
\hline ZA & - & + & - & - & - & - & - & - & - & - & - & - & - & - & - & - & - & - & - & - & - \\
\hline ZB & - & + & - & - & - & - & - & + & - & - & - & - & - & - & - & + & - & + & - & - & + \\
\hline $\mathrm{ZC}$ & - & - & - & - & - & - & - & + & - & - & - & - & - & - & - & - & - & - & - & - & - \\
\hline ZD & - & + & - & - & + & - & - & + & - & - & - & - & + & + & + & - & + & + & - & - & - \\
\hline $\mathrm{ZE}$ & - & + & - & - & - & - & - & - & - & - & - & - & - & - & - & - & - & - & - & - & - \\
\hline
\end{tabular}

Eiweißfraktionen (Tab. I): B - bei Streckmaden, $\mathrm{N}$ - bei Imagines (Abb. 1), L, O und $\mathrm{P}$ - bei Rundmaden, $\mathrm{X}$ und $\mathrm{Y}$ - bei dunkelund ZB, ZC und ZD - bei weissäugigen Puppen. Keine Fraktion des Darmextrakts erscheint in allen Stadien der postembryonalen Entwicklung.

Im Elektrophoresespektrum des Darmextrakts entdeckten wir keine über den Verlauf der gesamten postembryonalen
Entwicklung beständige Synthese, wie zum Beispiel bei der Fraktion V im Spektrum des Herzextrakts. So fehlt Fraktion V beim Puppenstadium im Darmextrakt.

\subsection{Hoden}

Im Verlauf der Ontogenese ist das Elektrophoresespektrum der Hoden bedeutend 


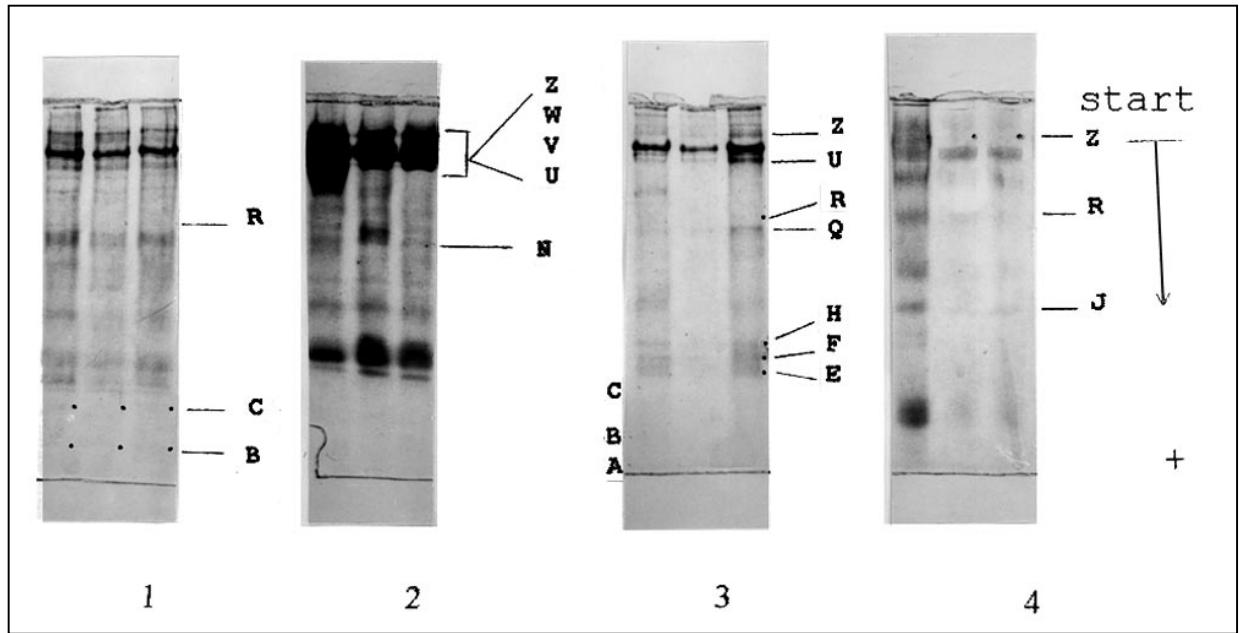

Abb. 1. Wasserlösliche Proteine bei Imago: 1) Herzextrakt; 2) Darmextrakt; 3) Mucusdrüsenextrakt; 4) Augenextrakt.

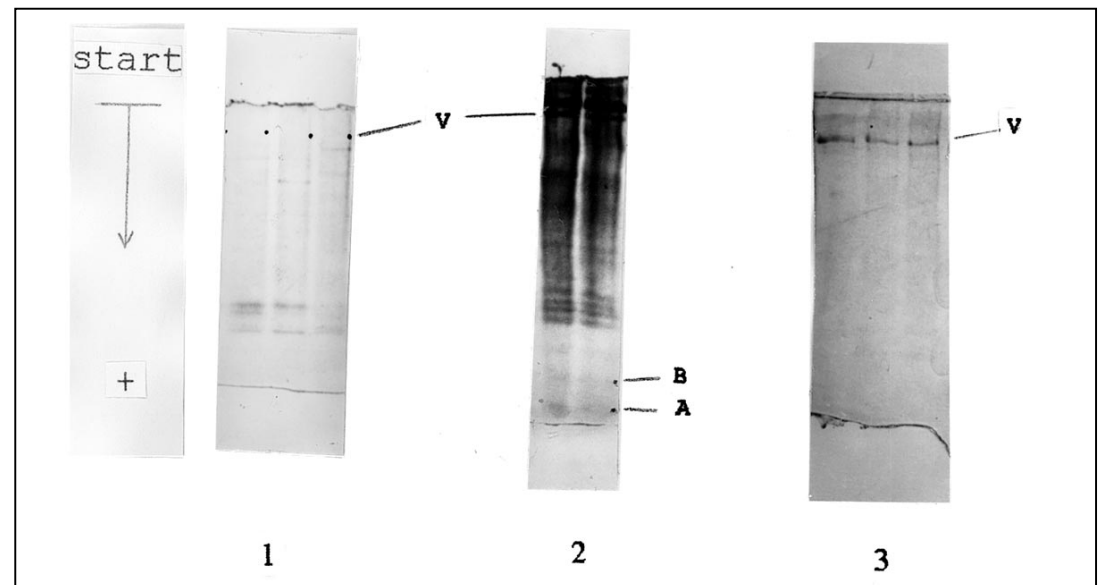

Abb. 2. Wasserlösliche Proteine im Hodenextrakt bei Drohnenontogenese: 1) Rundmaden; 2) Streckmaden; 3) Imago.

vielseitiger. Die Dynamik des Erscheinens der Fraktionen ist offensichtlich (Abb. 2, Tab. I) und kompliziert. So erscheinen die Fraktionen I, M, N, O und W während des Vorpuppenund Puppenstadiums, fehlen aber im Larvenund Imaginalstadium. Das Auftreten der Fraktionen $\mathrm{T}$ und $\mathrm{U}$ ist für die Periode von Rundmaden bis zu dunkeläugigen Puppen, das der Fraktionen J und Z von Streckmaden bis zu Imago charakteristisch.

Spezifisch für die Vesiculae seminales sind die Fraktionen A, B, F und G im Vorpuppenstadium.

Wie im Herzextrakt, so stellten wir auch hier Beständigkeit beim Erscheinen der 
Fraktion V im Verlauf der gesamten postembryonalen Periode fest (Tab. I).

\subsection{Mucusdrüse}

Die Elektrophoresedaten der Mucusdrüse zeigen, dass die Anzahl der Fraktionen vom Beginn der Verpuppung bis zur Imago wächst (Tab. I).

Eine Stadienspezifität des Auftretens ergab sich für die Fraktionen A, B, C, E, F, $\mathrm{H}, \mathrm{Q}, \mathrm{R}, \mathrm{U}$ und $\mathrm{Z}$ beim Imago (Abb. 1) und für die I - Fraktion bei dunkeläugigen Puppen. Für beide Entwicklungsperioden ist ein ständiges Erscheinen der W-Fraktion charakteristisch.

\subsection{Augen}

Das elektrophoretische Bild des Augenextrakts der Drohnen ist vielseitig bei Puppen und adulten Tieren (Abb. 1, Tab. I). Stadienspezifisch erscheinen die Fraktionen $\mathrm{C}$ - bei weissäugigen Puppen, $\mathrm{W}$ - bei dunkeläugigen Puppen und I,P,R,T,U und ZB bei Imagines. Die J und Z - Proteinfraktionen sind ständig vorhanden..

Insgesamt läßt sich feststellen, dass die 31 nachgewiesenen Eiweißfraktionen in keinem einzigen der untersuchten Organe im Verlauf der Individualentwicklung gleichzeitig auftreten. Die Anzahl der Fraktionen in den Organen ist unterschiedlich: im Herzextrakt fanden wir 22, im Darm 24, im Hodenextrakt 21, im Extrakt der Mucusdrüse 20 und im Augenextrakt 16 Fraktionen.

Die sich während der Individualentwicklung der Bienen abspielende Histolyse und Histogenese beeinflussen offensichtlich wesentlich die jeweilige Zusammensetzung des Eiweißspektrums.

\section{DISKUSSION}

Mit der Methode der nativen Elektrophorese in Polyacrylamidgel kann eine
Stadien- und Organspezifität des Erscheinens der wasserlöslichen Proteine bei den männlichen Formen der Honigbiene festgestellt werden. Außer der beschriebenen Stadiendynamik und Spezifität des Auftretens der wasserlöslichen Proteine bieten uns die erhaltenen Daten die Möglichkeit, auch organspezifisches Erscheinen festzustellen. So ist zum Beispiel die Fraktion A nur für das Geschlechtssystem der Drohnen charakteristisch, wir fanden sie in den Hoden und der Mucusdrüse, die Fraktion G nur in den Hoden.

Die Fraktionen K,L und ZC wurden im Darmextrakt nachgewiesen, ZA und ZE im Herzextrakt (Tab. I, Abb. 1 und 2).

Diese Ergebnisse weisen demnach vergleichbare Änderungen auf, wie sie bei früheren Untersuchungen über die Dynamik der Ausprägung wasserlöslichen Eiweiße im Verlauf der Individualentwicklung der Honigbienen in Haemolymphe und Totalextrakt auftraten.

Summary - Organ specificity of watersoluble proteins during drone (Apis mellifera L.) ontogenesis. Using polyacrylamide gel electrophoresis (PAGE), 368 organ extracts of drones were investigated. The soluble proteins spectra were analyzed in separate organs from the different specimens at various ontogenetic stages (unsealed larvae, sealed larvae, white-eyed pupae, dark-eyed pupae and imago forms). The organs tested (heart, gut, testicle, mucus glands and eyes) were isolated by dissection, rinsed with distilled water, squashed in $0.1 \mathrm{M}$ tris-phosphate buffer at $\mathrm{pH} \mathrm{6.7,}$ and left for extraction for $24 \mathrm{~h}$ at $4{ }^{\circ} \mathrm{C}$. Then the samples were centrifuged for $30 \mathrm{~min}$ at 900 g. For electrophoretic separation, a 7.5\% polyacrylamide vertical gel ( $\mathrm{pH} 8.9)$ was used, together with $3.3 \%$ concentrating gel at $\mathrm{pH} 6.7$ and $0.05 \mathrm{M}$ tris $-0.2 \mathrm{M}$ glycine electrode buffer, at $\mathrm{pH} 8.3$. The soluble proteins were visualised with Commassie Brilliant Blue R 250. 
A total of 31 fractions of water-soluble proteins were found in the spectra of analyzed organs -22 in heart extract, 24 in extract of gut, 21 in extracts of testes, 20 - in extracts of mucus glands and 16 - in eye extracts (Tab. I). An organ and stage specificity in the appearance of soluble proteins during development of Apis mellifera drones could be shown. Organ specific bands were found in the reproductive organs: testis ((A and $\mathrm{G})$ and mucus glands (A), as well as in the intestine $(\mathrm{K}, \mathrm{L}$ and $\mathrm{ZC})$ and the heart $(\mathrm{ZA}+\mathrm{ZE})$ (Figs. 1 and 2).

\section{Apis mellifera / drone / ontogenesis / water-soluble proteins}

Résumé - Étude par électrophorèse de la spécificité des protéines hydrosolubles visà-vis des organes au cours de l'ontogenèse du mâle d'abeille (Apis mellifera L.). On a analysé par électrophorèse sur gel de polyacrylamide (PAGE) les spectres de protéines hydrosolubles de 368 extraits d'organes de mâles d'abeille. Les organes (cœur, tube digestif, glandes à mucus, testicules et yeux) ont été étudiés à divers stades de développement : larves de 5 jours non operculées, larves de 7 jours operculées, nymphes de 3 jours aux yeux blancs, larves de 7 jours aux yeux noirs et mâles adultes âgés de 10 jours. Les organes ont été isolés par dissection, rincés à l'eau distillée, extraits pendant $24 \mathrm{~h}$ dans un tampon tris-phosphate à $0,1 \mathrm{M}(\mathrm{pH} 6,7)$, à la température de $4{ }^{\circ} \mathrm{C}$. Les échantillons ont été ensuite centrifugés pendant 30 minutes à $900 \mathrm{~g}$. La séparation par électrophorèse a été faite sur un gel vertical de polyacrylamide à $7,5 \%(\mathrm{pH} 8,9)$ et un gel de concentration à 3,3\%( $\mathrm{pH} 6,7)$, avec un tampon tris $0,05 \mathrm{M}-$ glycine $0,2 \mathrm{M}$ à l'anode et la cathode ( $\mathrm{pH} 8,3)$. Les protéines ont été visualisées avec le bleu Brillant de Commassie R250.

Au total 31 fractions de protéines hydrosolubles ont été trouvées dans les extraits : 22 dans le cœur, 24 dans le tube digestif, 21 dans les testicules, 20 dans les glandes à mucus et 16 dans les yeux (Tab. I). On a pu montrer une spécificité des protéines vis-àvis des organes et des stades de développement. Des bandes spécifiques des organes ont été trouvées dans les extraits des organes de reproduction (testicules : A et $\mathrm{G}$, glandes à mucus : A) ainsi que dans ceux du tube digestif (K, $\mathrm{L}$ et $\mathrm{CZ}$ ) et du cœur (ZA et $\mathrm{ZE})$ (Figs. 1 et 2).

\section{Apis mellifera / protéine hydrosoluble / ontogenèse / mâle}

\section{LITERATUR}

[1] Arbuthnot P.B., Cantill R.C., Herbum H.R., An electrophoretic characterization of African worker honey bee hemolymph proteins during development, Comp. Biochem. Physiol. B 74 (1983) 467-471.

[2] Danty E., Arnold G., Burmester T., Huet J.C., Huet D., Pernollet J.C., Masson C., Identification and developmental profiles of hexamerins in antenna and hemolymph of the honeybee, Apis mellifera, Insect Biochem. Mol. Biol. 28 (1998) 387-397.

[3] Engels W., Fahrenhorst H., Age-dependent and caste-dependent changes in hemolymph protein patterns of Apis mellifera, Wilhelm Roux Arch. Entwickl. Mech. Org. 174 (1974) 285-296.

[4] Glinski Z., Jarosz J., Polyacrylamide gel electrophoresis in observations on insect haemolymph, Med. Wet. 3 (1986) 159-164 (in Polish).

[5] Glinski Z., Jarosz J., Werniski A., An immunoelectrophoretical analysis of soluble blood proteins of coiled and upright larvae of the worker honey bee, Apis mellifera, Apidologie 16 (1985) 247-265.

[6] Huang Z.Y., Otis G.W., Teal P.E.A., Nature of brood signal activating the protein synthesis of hypopharyngeal gland in honey bees, Apis mellifera (Apidae, Hymenoptera), Apidologie 20 (1989) 455-464.

[7] Ivanova E., Izsledvanija varchu elektroforeticnite spektri na raztvorimi beltaci pri Apis mellifera L., Plovdiv University, Trav. Sci. 29 (1991) 247-250.

[8] Krieg P., Marek M., Protein and esterase changes in the hemolymph of the queens of honey bee, Apis mellifera L., Comp. Biochem. Physiol. B-Biochem. and Mol. Biol. 75 (1983) 513-517.

[9] Kubo T., Sasaki M., Nakamura J., Sasagawa H., Ohashi K., Takeushi H., Natori S., Change in the expression of hypopharyngeal gland proteins of the worker honeybees (Apis mellifera L.) with age and /or role, J. Biochem. 119 (1996) 291-295. 
[10] Maurer G., Disk-elektroforez, Mir, Moskva, 1971.

[11] Michelette E., Engels W., Concentration of hemolymph proteins during postembryonic worker development of Africanized honey bees in Brazil and Carniolans in Europe, Apidologie 26 (1995) 101-108.

[12] Ohashi K., Natori S., Kubo T., Change in the mode of gene expression of the hypopharyngeal gland cells with an age-dependent role change of the worker honeybee Apis mellifera L., Eur. J. Biochem. 249 (1997) 797-802.

[13] Ohashi K., Sawata M., Takeuchi H., Natori S., Kubo T., Molecular cloning of cDNA and analysis of expression of the gene far alpha- glucosidase from the hypopharyngeal gland of the honeybee Apis mellifera L., Biochem. Biophys. Res. Commun. 221 (1996) 380-385

[14] Popov P., Ivanova E., Electrophoretic studies on the total soluble protein in imago forms of Apis mellifera L., Animalia 30 (1994) 53-56.

[15] Raolison C., Sutter B., An intestinal insulin-like molecule in Apis mellifera, Comp. Biochem. Physiol. A 69 (1981) 79-83.

[16] Shao-Wen Li, Jang Qwen Mei, Meng Jo-Pin, Chang I., Studies on the haemolymph protein pattern of two species of honey bees Apis mellifera, Acta Entomol. Sin. 25 (1982) 185-190 (in Chinese). 